\title{
Kawasaki Disease Shock Syndrome: A Challenging Diagnosis
}

\author{
Raquel Penteado ${ }^{\mathrm{a}, \mathrm{c}}$, Ana Sofia Simoes ${ }^{\mathrm{a}}$, Andreia Palma ${ }^{\mathrm{b}}$, \\ Joana Andrade ${ }^{a}$, Teresa Dionisio ${ }^{a}$
}

\begin{abstract}
The authors present a clinical report of a previously healthy 4-yearold girl, admitted to a pediatric intensive care unit (PICU) presenting with a fluid-refractory shock. She was initially admitted in the pediatric emergency department with a history of fever and petechial rash and an initial diagnosis of invasive meningococcal disease was placed. During the PICU stay, acute myocarditis with episodes of supraventricular tachycardia and lateral and inferior wall myocardial ischemia, ileitis and serositis were noted. At 14th day of stay, she developed peeling on the hands and feet and, aneurysmatic ectasia of the coronary arteries were visualized in transthoracic echocardiography, allowing the diagnosis of Kawasaki disease shock syndrome (KDSS). The child improved after empirical treatment with antibiotics, intravenous immunoglobulin and corticosteroid therapy. She was started on antiplatelet therapy due to changes in the coronary arteries. Early recognition of KDSS can be challenging; however, a delayed diagnosis increases the risk of coronary changes and a fatal outcome.
\end{abstract}

Keywords: Kawasaki disease shock syndrome; Kawasaki disease; Shock

\section{Introduction}

Kawasaki disease (KD) is the most common pediatric cause of acquired heart disease in developmental countries and can lead to permanent changes in the coronary arteries in up to $25 \%$ of untreated cases. However, initial presentation with hemodynamic instability is not common. KD shock syndrome (KDSS)

Manuscript submitted December 11, 2020, accepted December 30, 2020

Published online March 19, 2021

aPediatric Intensive Care Service, Hospital Pediatrico, Centro Hospitalar e Universitario de Coimbra, Portugal

${ }^{b}$ Cardiology Service, Hospital Pediatrico, Centro Hospitalar e Universitario de Coimbra, Portugal

${ }^{\mathrm{c} C o r r e s p o n d i n g ~ A u t h o r: ~ R a q u e l ~ P e n t e a d o, ~ S e r v i c o ~ d e ~ C u i d a d o s ~ I n t e n s i v o s, ~}$ Hospital Pediatrico, Centro Hospitalar e Universitario de Coimbra, Avenida, R. Dr. Afonso Romao, 3000-602 Coimbra, Portugal.

Email: rtcpenteado@gmail.com

doi: https://doi.org/10.14740/ijcp425 was first described in $2009[1,2]$. The cause of severe KDSS hypotension is not yet fully known, although it is believed to be due to vasculitis along with capillary leakage, myocardial dysfunction and generalized cytokine dysregulation. KDSS is an under-diagnosed entity, with an estimated occurrence in $5-7 \%$ of children with KD [2-4].

As KDSS is a more severe form of $\mathrm{KD}$, the delay in diagnosis contributes to a longer hospitalization and higher morbidity and mortality. KDSS should be considered in all children with a refractory shock admitted to pediatric intensive care unit (PICU) who do not improve with antibiotic treatment and whose cultures remain negative, even if they do not initially meet the criteria for $\mathrm{KD}$ [3]. In this particular case report, the diagnosis of KDSS was made at a late stage of the disease. The development of skin peeling on the hands and feet led us to perform a transthoracic echocardiogram, which showed coronary aneurysms and thus confirming the KDSS diagnosis. A second dose of intravenous immunoglobulin (IGIV) was administered, after which a good evolution of the patient's condition was noted.

\section{Case Report}

A 4-year-old girl presented to the emergency department (ED) with a 4-day history of fever, cough, rhinorrhea, abdominal pain, vomiting, and pain and edema in the lower limbs. She was on day 4 of amoxicillin/clavulanic acid $(80 \mathrm{mg} / \mathrm{kg} /$ day $)$ due to an acute otitis media previously diagnosed in primary care outpatient clinic. On admission, she was feverish (axillary temperature $39.5^{\circ} \mathrm{C}$ ) and intermittently grunting. On physical examination, she showed moderate signs of respiratory distress, peripheral oxygen saturation level of $95 \%$ in room air, heart rate of 110 beats per minute, blood pressure of 99/65 $\mathrm{mm} \mathrm{Hg}$ and a capillary refill time below $2 \mathrm{~s}$. Additionally, she presented a macular rash on her hands and face. Neurological examination findings were unremarkable including negative signs of meningeal irritation.

The national immunization programme was updated with three doses of 13-valent pneumococcal conjugate vaccine. Past medical, social, environmental and family history were all unremarkable.

The initial evaluation showed leucocytosis of $20,750 / \mu \mathrm{L}$ with neutrophilia (90\%) and C-reactive protein (CRP) 28.97 $\mathrm{mg} / \mathrm{dL}$; chest radiography showed a left paracardiac infiltrate. 


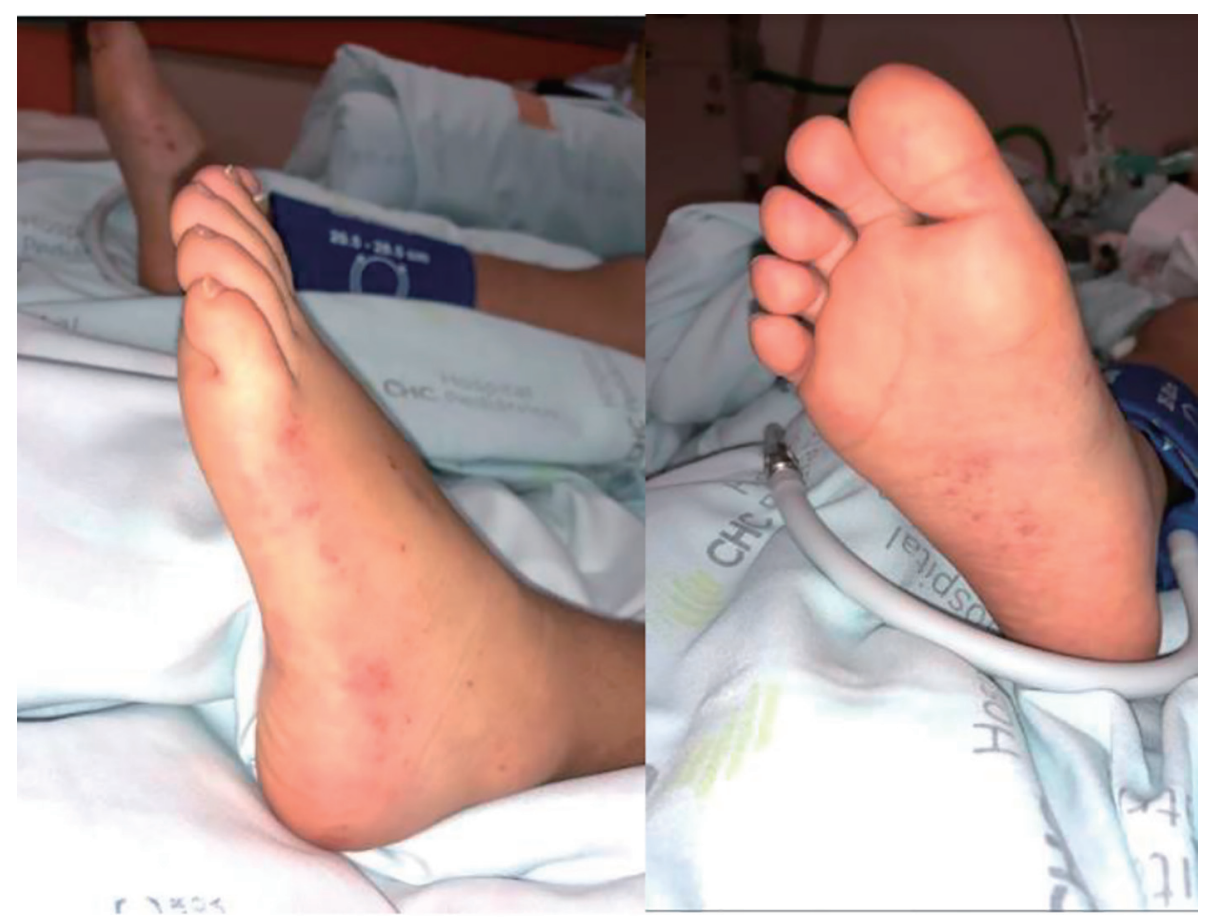

Figure 1. Rash of the lower limbs.

Parainfluenza 1, rhinovirus/enterovirus and adenovirus were identified in respiratory secretions by polymerase chain reaction (PCR).

She was admitted to a pediatric ward with a diagnosis of pneumonia and started on ceftriaxone $80 \mathrm{mg} / \mathrm{kg} / \mathrm{day}$. In the first hours of hospitalization, due to a change in hemodynamic profile (hypotension, with blood pressure 68/35 $\mathrm{mm} \mathrm{Hg}$ ) and altered state of consciousness, between drowsiness and psychomotor agitation, normal saline bolus was administered and brain computed tomography (CT) scan was performed (no abnormalities reported). On the same day, petechial lesions and suffusions in both lower limbs appeared (Fig. 1). Lumbar puncture was postponed. The dose of ceftriaxone was increased to $100 \mathrm{mg} / \mathrm{kg} /$ day owing to the suspicion of meningococcemia, and acyclovir (10 mg/kg IV q8h) and ciprofloxacin $(10 \mathrm{mg} / \mathrm{kg}$ IV q12h) were initiated. She was transferred to a PICU by a medical transport team.

On day 1 of admission to the PICU, her clinical state deteriorated. She showed a decreased state of consciousness with a persistently low Glasgow coma scale (GCS) score of 8 - 10, and a decompensated shock with a heart rate of 130 beats per minute, a blood pressure of $68 / 41 \mathrm{~mm} \mathrm{Hg}$ and an immediate capillary refill time. She was invasively ventilated for the first 12 days (maximum positive end-expiratory pressure (PEEP) 7 $\mathrm{mm} \mathrm{Hg}$ ), and required oxygen until day 18 of hospitalization $\left(\mathrm{FiO}_{2}\right.$ max 60\%). On the fourth day, a $3 \mathrm{~cm}$ anechoic bilateral pleural effusion, lung atelectasis and significant bilateral interstitial syndrome were visible in pulmonary ultrasound scan (Fig. 2).

She required cardiovascular support with dopamine (maximum $10 \mu \mathrm{g} / \mathrm{kg} / \mathrm{min}$ ), adrenaline (maximum $0.4 \mu \mathrm{g} / \mathrm{kg} / \mathrm{min}$ ) and norepinephrine (maximum $0.5 \mu \mathrm{g} / \mathrm{kg} / \mathrm{min}$ ), with gradual reduction until day 13 . She was on hydrocortisone until day 4. On day 3 , the patient developed bilateral conjunctival hyperemia.

Both incomplete KD (unexplained prolonged fever, bilateral conjunctival hyperemia, and erythema and edema of the hands and feet) and autoimmune disease (unexplained prolonged fever, serositis and high analytical inflammatory parameters) were hypothesized and IVIG ( $1 \mathrm{~g} / \mathrm{kg}$ ) was administered.

Echocardiogram previously performed showed no structural abnormalities. On day 5 impairment of ventricular left dysfunction was identified, with an ejection fraction (EF) of $23 \%$ along with junctional rhythm on electrocardiogram (ECG). Between days 7 and 8, multiples episodes of supraventricular tachycardia occurred, two of which with hemodynamic instability resolved after IV adenosine administration. Still on day 8, an ECG recorded ST depression (V3-V5). From day 8 on, there was progressive improvement in cardiac function and reduction in high-sensitivity cardiac troponin test (maximum $404 \mathrm{ng} / \mathrm{mL}$ on day 3 , normal $>16 \mathrm{ng} / \mathrm{mL}$ ), and N-terminal pro B-type natriuretic peptide (NT-proBNP) (maximum $>35,000$ $\mathrm{pg} / \mathrm{mL}$ on day 3 ). Myocarditis was then diagnosed.

Palmar desquamation was observed on day 14, which led to an echocardiogram where changes were detected in the coronary arteries with a $5 \mathrm{~mm}$ aneurysmatic dilatation at the beginning of the right coronary artery and rosary aneurysmatic dilatation on the left (Figs. 3 and 4). As KD was confirmed (with coronary involvement), the patient had a new administration of IVIG $(2 \mathrm{~g} / \mathrm{kg})$ and was put on double antiplatelet therapy with acetylsalicylic acid (initial dose of $40 \mathrm{mg} / \mathrm{kg} /$ day and maintenance dose of $5 \mathrm{mg} / \mathrm{kg} /$ day $)$ and clopidogrel $(0.21 \mathrm{mg} / \mathrm{kg} /$ day) and anticoagulation therapy with enoxaparin $(1 \mathrm{mg} / \mathrm{kg} /$ 


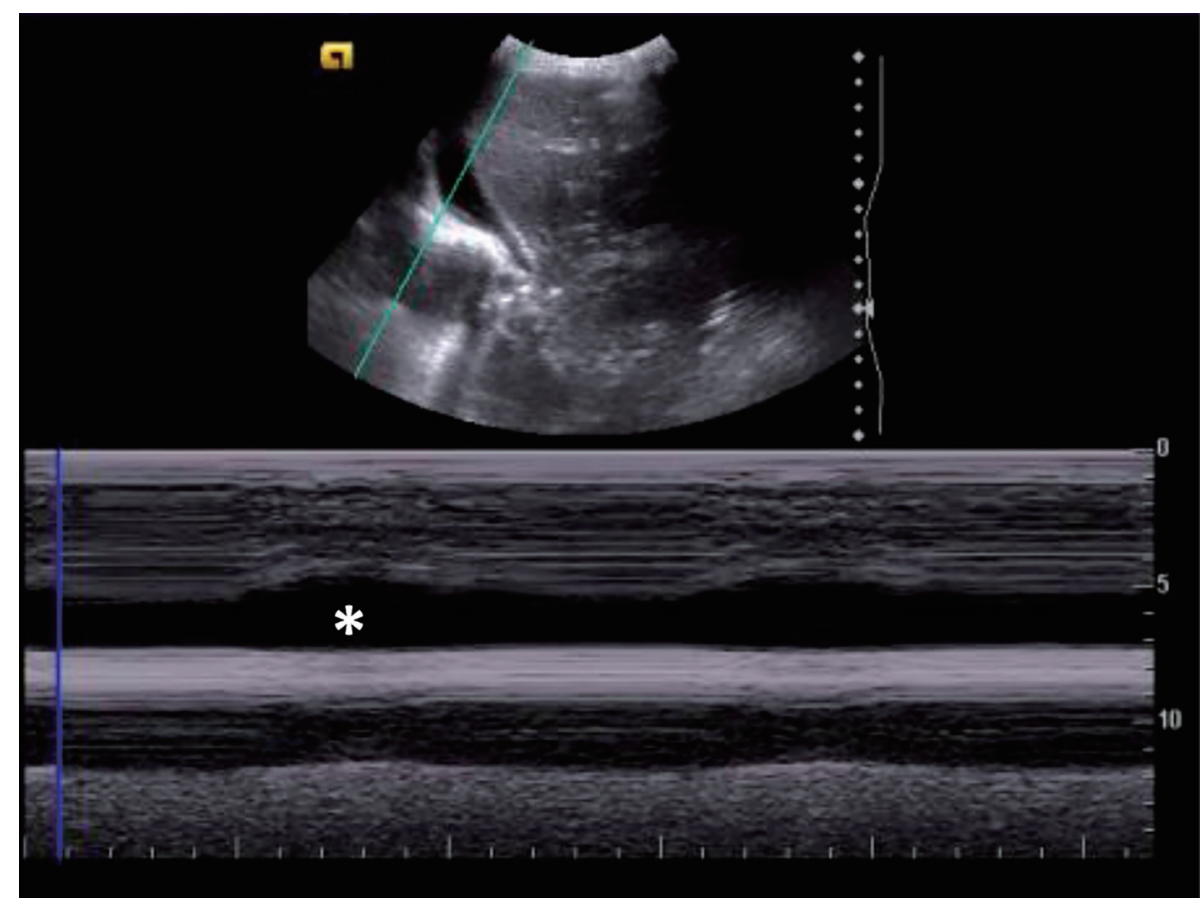

Figure 2. Pulmonary ultrasound showing anechogenic pleural effusion with sinusoid sign (M-mode). The sinusoid sign $\left({ }^{*}\right)$ is a dynamic sonographic sign, present when respiratory variation decreases the distance between the parietal and visceral pleura, when separated by a pleural effusion.

day). We proceeded with serial echocardiogram evaluation and on day 23 , normal origin coronary arteries were observed on the echocardiogram, with a common trunk of $3.7 \mathrm{~mm}$ (z-score +4.2 ) (Fig. 5), anterior descending of $4.0 \mathrm{~mm}$ (z-score +6.7$)$, circumflex of $2.3 \mathrm{~mm}(\mathrm{z}$-score +1.9$)$ and right coronary 4.1 $\mathrm{mm}(\mathrm{z}$-score +5.7$)$; the 12-lead ECG maintained changes in repolarization with negative T wave in DI, DII, aVL and V4-V6.

Acute kidney injury with initial oliguria was present. Re-

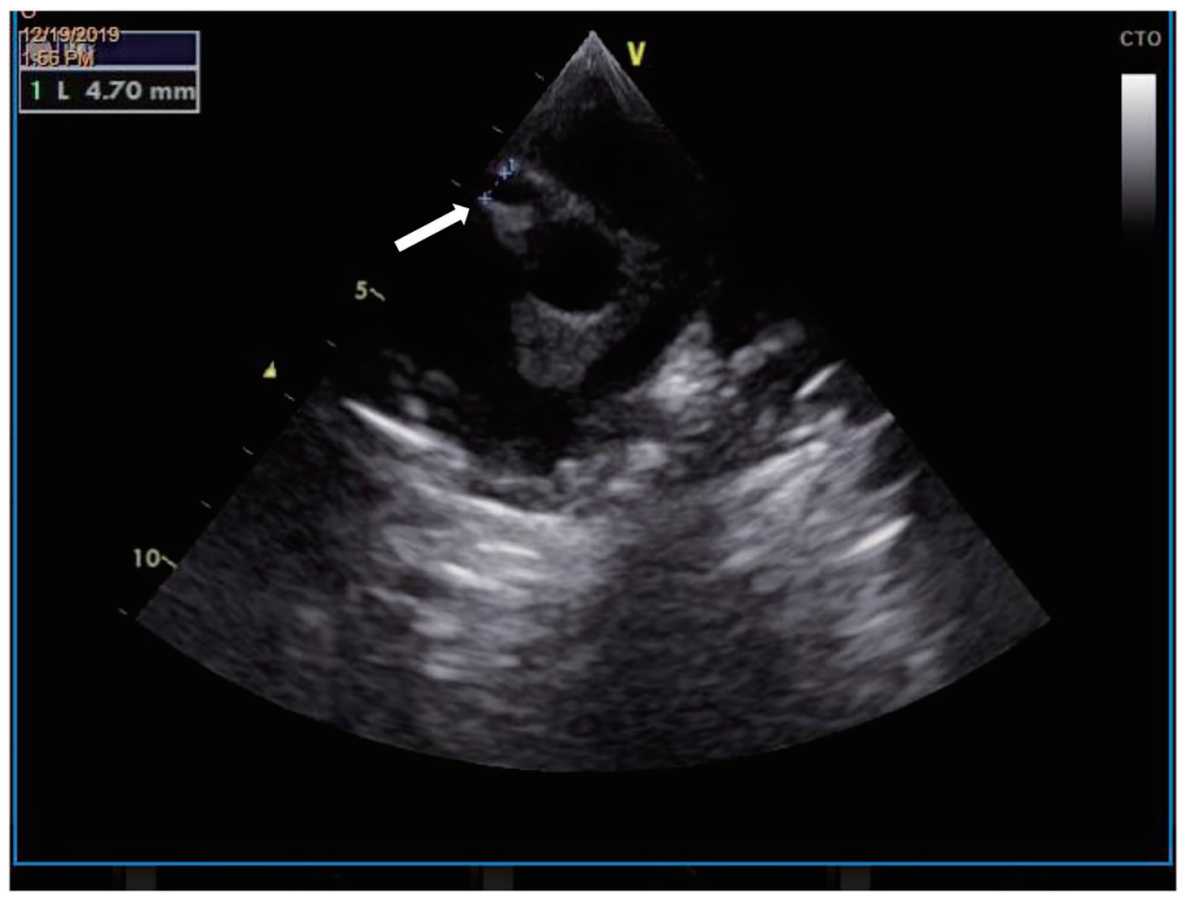

Figure 3. Transthoracic echocardiography demonstrating an aneurysmatic dilatation $(4.7 \mathrm{~mm})$ at the beginning of the right coronary artery (white arrow). 


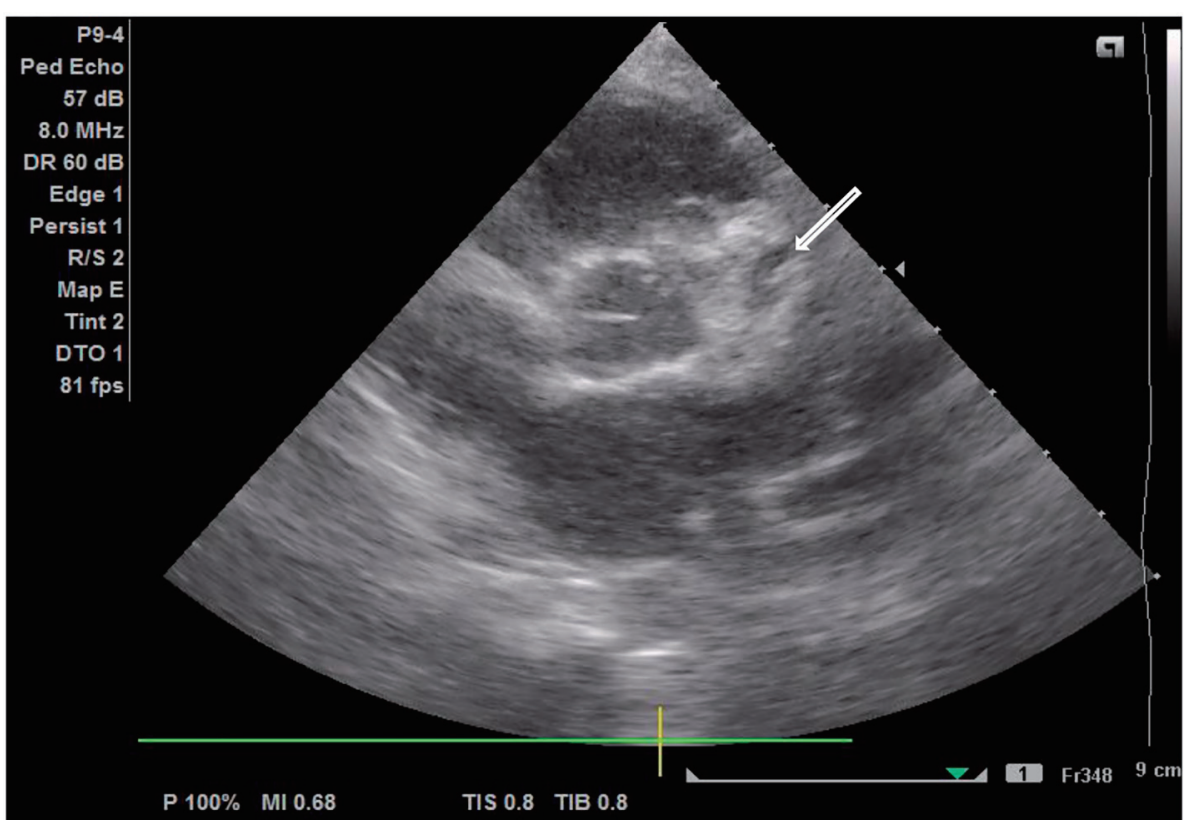

Figure 4. Transthoracic echocardiography demonstrating a rosary aneurysmatic dilatation of the left coronary artery (white arrow).

covery ensued after hemodynamic optimization. Lower limb edema was progressively better, associated with improvement of hypoalbuminemia (minimum $21 \mathrm{U} / \mathrm{L}$ on first day).

On the first day, because of abdominal distension, an abdominal ultrasound was performed, which revealed thickening of distal ileon with adjacent adenomegalies (Fig. 6). As a result of progressive worsening of the abdominal pain and distension, abdominal CT was executed (day 15). These confirmed the presence of moderate free peritoneal effusion and liquid distention of the ileal loops, with no thickening of the intestinal walls nor strictures. Laboratory results showed elevated transaminases levels (maximum glutamic oxaloacetic transaminase (GOT) $537 \mathrm{U} / \mathrm{L}$ and glutamic pyruvic transaminase (GPT) $144 \mathrm{U} / \mathrm{L}$ ).

During the PICU stay, the patient maintained persistent fever until day 8 (total of 12 days of fever). Analytical assessment showed maximum leukocyte values and inflammatory parameters registered on day 3: leucocytosis of $34,270 / \mu \mathrm{L}$ (89\% neutrophils), CRP $30 \mathrm{mg} / \mathrm{dL}$ and procalcitonin $310 \mathrm{ng} /$ $\mathrm{mL}$ (Table 1).

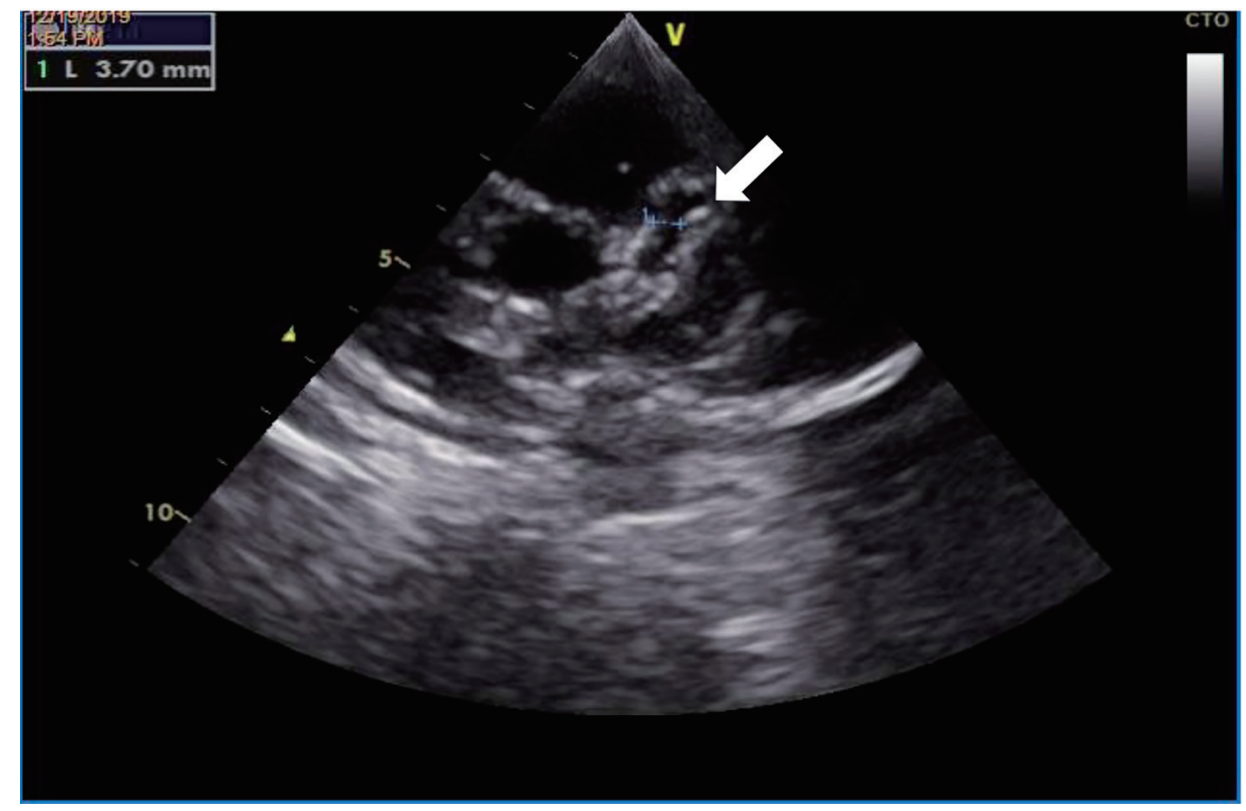

Figure 5. Transthoracic echocardiography demonstrating a dilatation of left main coronary artery (3.7 mm) 


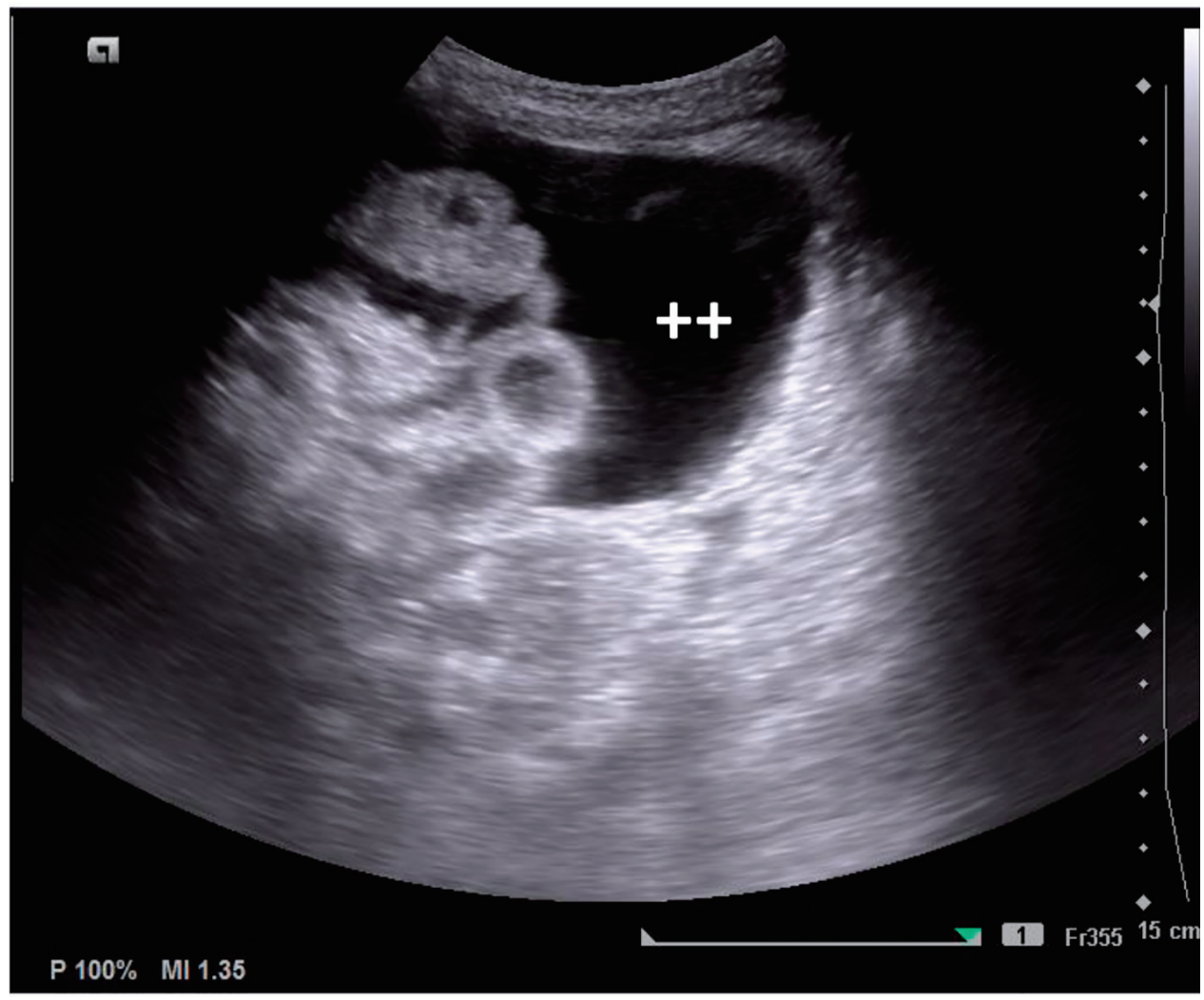

Figure 6. Abdominal ultrasound, with thickening of the terminal ileum and large amount of peritoneal effusion (++).

Table 1. Summary of Blood Results During Inpatient Admission

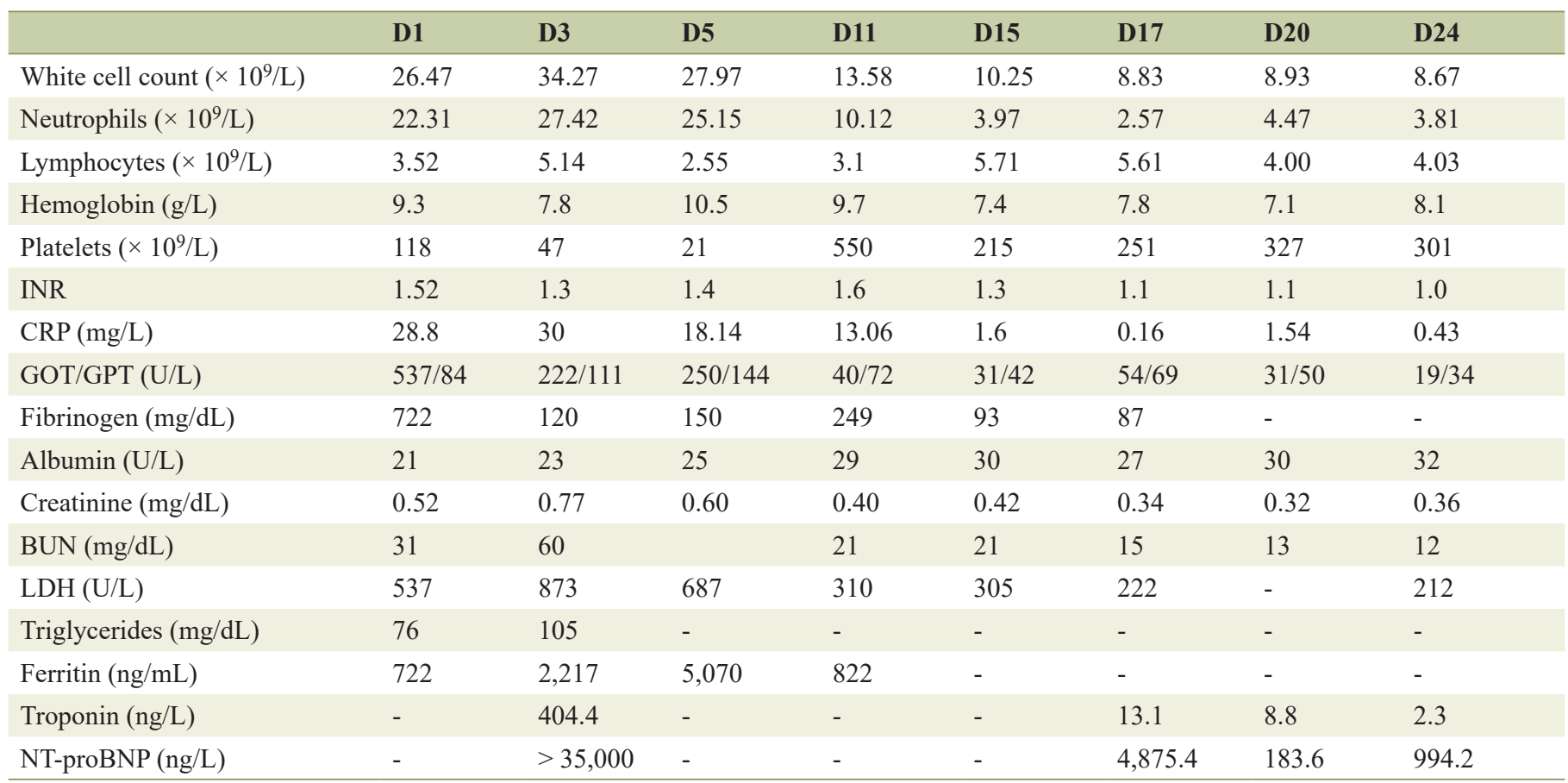

BUN: blood urea nitrogen; CRP: C-reactive protein; GOT: glutamic oxaloacetic transaminase; GPT: serum glutamic pyruvic transaminase; INR: international normalized ratio; LDH: lactate dehydrogenase; NT-proBNP: N-terminal pro B-type natriuretic peptide. 
The main infectious causes were ruled out: serologic tests for brucellosis, cytomegalovirus, herpes simplex virus 1 and 2, Epstein-Barr virus, Mycoplasma pneumoniae; plasma PCR for meningococcus, pneumococcus, enterovirus, parvovirus B19, adenovirus; multiplex PCR rectal swab including Salmonella, Yersinia, adenovirus, norovirus; blood cultures (central and peripheral-blood cultures), urine culture, stool cultures and bone marrow aspirate culture, which were all negative (for aerobic, anaerobic and fungi). Serological tests for specific SARS-CoV-2 antibodies (IgM/IgG) were negative.

On day 1 of admission to the PICU, empiric antimicrobial therapy with vancomycin and metronidazole was started, both to cover for Gram-positive and anaerobic bacteria. Acyclovir and ceftriaxone were suspended on day 2. Due to an unexplained prolonged fever, raised inflammatory parameters and an increased abdominal distension, empiric treatment with meropenem was initiated to cover for a potential intraabdominal infection. She completed a total of 19 days of vancomycin (20 mg/kg IV q12h), 16 days of ciprofloxacin $(10 \mathrm{mg} / \mathrm{kg} \mathrm{IV}$ q12h), 15 days of meropenem ( $20 \mathrm{mg} / \mathrm{kg}$ IV q12h) and 7 days of metronidazole $(7.5 \mathrm{mg} / \mathrm{kg}$ IV q8h). She was put on methylprednisolone $(1 \mathrm{mg} / \mathrm{kg} /$ day $)$ on day 4 , due to the suspicion of hemophagocitic syndrome, which was changed on day 17 to prednisolone (1 mg/kg PO q12h), until day 24, after gradual reduction.

Several blood analyses were performed, showing anemia (minimum $\mathrm{Hb} 6.8 \mathrm{~g} / \mathrm{dL}$ ) and thrombocytopenia (minimum of $12,000 / \mu \mathrm{L}$ platelets). Minimum fibrinogen assay was $87 \mathrm{~g} /$ $\mathrm{dL}$, and maximum ferritin was 5,070 ng/mL. Furthermore, on suspicion of secondary macrophage activation syndrome (prolonged fever, rash, hypofibrinogenemia, hyperferritinemia and bicytopenia), a medullogram was performed on day 5 , which could suggest reactive neutropoiesis, without hemophagocytosis. A transfusion of red blood cells was given on day 4 for anemia and platelet transfusion for thrombocytopenia on day 5 , day 8 and day 9 .

\section{Outcome and follow-up}

The patient was transferred to her local hospital 25 days after admission to the PICU, where she was hospitalized for another 18 days. Another echocardiogram was performed before discharge with similar findings and a 12-lead ECG was normal. She underwent a programme of rehabilitation due to reduced muscle strength in the lower limbs, and made a good recovery. She maintained prednisolone, acetylsalicylic acid and clopidogrel as home therapy with gradual reduction.

She is currently being followed up in the cardiology and rheumatology consultation. Nine months after being discharged, she is asymptomatic. In the last echocardiogram, no ectasias or other alterations were visible and only therapy with acetylsalicylic acid is maintained.

\section{Discussion}

In our case report, a previously healthy 4-year-old girl present- ed prolonged fever, erythema and edema of the lower limbs, bilateral conjunctival injection, refractory shock, hypoalbuminemia, myocardial dysfunction with tachyarrhythmia, marked thrombocytopenia, higher levels of CRP and ileitis. Despite the clinical signs, the diagnosis of KDSS was only confirmed at a later stage of the disease after coronary aneurysms were recorded in the transthoracic echocardiogram. These followed the observation of skin peeling on the hands and feet.

KDSS was defined in 2009 by Kanegaye et al by the presence of characteristics of KD and one of the following: systolic hypotension (systolic blood pressure below -2SD according to sex and age) and over $20 \%$ decrease in blood pressure baseline or signs of poor perfusion.

KDSS is a multisystemic disease, with cardiac, gastrointestinal, respiratory, renal and sometimes neurological manifestations [3]. Although the etiology is still not well defined, it is believed that severe vasculitis with increased vascular permeability as well as myocardial dysfunction can lead to cytokine dysregulation, which results in cardiogenic and/or distributive shock [5].

In the largest study conducted with patients with KDSS, cases were prevalent in the winter months and in children over 5 years of age (with peak incidence between 8 and 9 years of age) [4].

When compared to KD, KDSS has been shown to occur more frequently in female patients, whom initially present an incomplete clinical picture of KD along with more serious analytical changes, namely marked thrombocytopenia, higher levels of CRP, elevated transaminases and increased risk of cardiac involvement $[3,6]$. These characteristics are all in line with the clinical case presented.

The literature describes cases of KDSS in which hypoalbuminemia with pleural effusion, pericardial effusion and ascites may be present. In most patients, there also seems to appear an association with previous abdominal pain and gastrointestinal symptoms, the severity of which can range from diarrhea and vomiting to bleeding and even intestinal perforation [3]. In our case, the girl presented a clinical status of shock on the fourth day of fever, associated with gastrointestinal symptoms (abdominal pain and vomiting), including ileitis and peritoneal effusion, pleural effusion and respiratory symptoms (cough and rhinorrhea).

Cardiovascular changes are also common in KDSS, with coronary aneurysms being present in about $66 \%$ of cases, as opposed to $25 \%$ in untreated $\mathrm{KD}$, as well as a higher incidence of valvular and/or myocardial dysfunction and inflammation (elevated NT-proBNP and troponin values and increased incidence of arrhythmias) $[1,3,7]$. In our case, she presented with myocardial dysfunction requiring vasoactive support, myocarditis with tachydysrhythmia and coronary aneurysms. Anti-aggregation therapy is in accordance with the recent guidelines for the treatment of KD [8].

During the patient's hospitalization, macrophage activation syndrome was suspected, which was in accordance with some cases described, where they occurred simultaneously [9, 10]. However in our case, it was not confirmed.

Attributable to the more severe course of the disease than $\mathrm{KD}$, there is evidence of resistance to IVIG. It is often necessary a second prescription of IVIG and corticosteroid therapy 
(ideally before IVIG) and, more rarely, additional therapies may be necessary, in addition to all supportive treatment $[2,3]$. In this case report, the patient received treatment with corticosteroids and first dose of IVIG on an early stage of the disease, when incomplete KD was thought. Despite this, it is worth noting the patient's prolonged hospitalization in this clinical case, longer than that described in the literature (average of $8-9$ days) $[4,11]$. This may be owing to the severe initial presentation, with significant cardiac and respiratory involvement and the need for prolonged invasive ventilation.

Delay in diagnosing KDSS has been described on account of the initial presentation with shock, often mistakenly diagnosed at this stage as septic shock, which in turn delays the start of treatment with IVIG, or even toxic shock syndrome [12]. As KDSS is a more severe form of KD, this delay in diagnosis contributes to a longer hospitalization, higher morbidity and mortality [3].

KDSS should be considered in children with shock/hypotension admitted to the intensive care unit who do not improve with antibiotic treatment and whose cultures (blood and urine) remain negative, even if they do not initially meet the criteria for KD. Early recognition and diagnosis of KDSS allows timely initiation of treatment, reducing the risk of coronary artery aneurysms $[3,12]$.

\section{Learning points}

1) KDSS should be considered as a differential diagnosis in children admitted to intensive care with shock/hypotension with no improvement after antibiotic treatment and whose cultures remain negative.

2) Serial echocardiograms are recommended in these cases in order to identify cardiac involvement on an early stage, particularly aneurysms.

3) Early diagnosis of KDSS is essential to start appropriate therapy and reduce the risk of coronary aneurysms, which occur more frequently in these cases.

\section{Acknowledgments}

None to declare.

\section{Financial Disclosure}

None to declare.

\section{Conflict of Interest}

None to declare.

\section{Informed Consent}

Informed consent was obtained.

\section{Author Contributions}

RP, ASS, AP, JA and TD participated in the management of the patient and gave substantial contributions to this work. RP wrote the manuscript with support from ASS and AP. Review and editing: JA and TD. Each author has given final approval of the final version.

\section{Data Availability}

The authors declare that data supporting the findings of this study are available within the article.

\section{References}

1. Kanegaye JT, Wilder MS, Molkara D, Frazer JR, Pancheri J, Tremoulet AH, Watson VE, et al. Recognition of a Kawasaki disease shock syndrome. Pediatrics. 2009;123(5):e783789.

2. Taddio A, Rossi ED, Monasta L, Pastore S, Tommasini A, Lepore L, Bronzetti G, et al. Describing Kawasaki shock syndrome: results from a retrospective study and literature review. Clin Rheumatol. 2017;36(1):223228.

3. Gamez-Gonzalez LB, Moribe-Quintero I, CisnerosCastolo M, Varela-Ortiz J, Munoz-Ramirez M, GarridoGarcia M, Yamazaki-Nakashimada M. Kawasaki disease shock syndrome: Unique and severe subtype of Kawasaki disease. Pediatr Int. 2018;60(9):781-790.

4. Lin MT, Fu CM, Huang SK, Huang SC, Wu MH. Population-based study of Kawasaki disease shock syndrome in Taiwan. Pediatr Infect Dis J. 2013;32(12):1384-1386.

5. Gamez-Gonzalez LB, Murata C, Munoz-Ramirez M, Yamazaki-Nakashimada M. Clinical manifestations associated with Kawasaki disease shock syndrome in Mexican children. Eur J Pediatr. 2013;172(3):337-342.

6. Dominguez SR, Friedman K, Seewald R, Anderson MS, Willis L, Glode MP. Kawasaki disease in a pediatric intensive care unit: a case-control study. Pediatrics. 2008;122(4):e786-790.

7. Ma L, Zhang YY, Yu HG. Clinical Manifestations of Kawasaki Disease Shock Syndrome. Clin Pediatr (Phila). 2018;57(4):428-435.

8. de Graeff N, Groot N, Ozen S, Eleftheriou D, Avcin T, Bader-Meunier B, Dolezalova P, et al. European consensus-based recommendations for the diagnosis and treatment of Kawasaki disease - the SHARE initiative. Rheumatology (Oxford). 2019;58(4):672-682.

9. Cummings C, McCarthy P, van Hoff J, Porter G, Jr. Kawasaki disease associated with reactive hemophagocytic lymphohistiocytosis. Pediatr Infect Dis J. 2008;27(12): 1116-1118.

10. Pilania RK, Arora K, Gupta A, Vignesh P, Suri D, Singh S. A 5-year-old boy with Kawasaki disease shock syndrome, myocarditis and macrophage activation syndrome. J Paediatr Child Health. 2020. 
11. Chen PS, Chi H, Huang FY, Peng CC, Chen MR, Chiu NC. Clinical manifestations of Kawasaki disease shock syndrome: a case-control study. J Microbiol Immunol Infect. 2015;48(1):43-50.
12. Camacho-Lovillo MS, Melon M, Moreno MD, Ardanuy AV, Neth O. Kawasaki Disease Shock Syndrome (KDSS) - presentation of 3 children and review of the literature. Klin Padiatr. 2015;227(6-7):355-357. 